\title{
The Koolen-de Vries syndrome: a phenotypic comparison of patients with a 17q21.31 microdeletion versus a KANSL1 sequence variant
}

\author{
David A Koolen ${ }^{\star}, 1$, Rolph Pfundt ${ }^{1}$, Katrin Linda ${ }^{1}$, Gea Beunders ${ }^{2}$, Hermine E Veenstra-Knol ${ }^{3}$, Jessie H Conta ${ }^{4}$, \\ Ana Maria Fortuna ${ }^{5}$, Gabriele Gillessen-Kaesbach ${ }^{6}$, Sarah Dugan ${ }^{7}$, Sara Halbach ${ }^{8}$, Omar A Abdul-Rahman ${ }^{9}$, \\ Heather M Winesett ${ }^{10}$, Wendy K Chung ${ }^{11}$, Marguerite Dalton ${ }^{12}$, Petia S Dimova ${ }^{13}$, Teresa Mattina ${ }^{14}$, \\ Katrina Prescott ${ }^{15}$, Hui Z Zhang ${ }^{16}$, Howard M Saal ${ }^{17}$, Jayne Y Hehir-Kwa ${ }^{1}$, Marjolein H Willemsen ${ }^{1}$, \\ Charlotte W Ockeloen ${ }^{1}$, Marjolijn C Jongmans ${ }^{1}$, Nathalie Van der Aa ${ }^{18}$, Pinella Failla ${ }^{19}$, Concetta Barone ${ }^{19}$, \\ Emanuela Avola $^{19}$, Alice S Brooks ${ }^{20}$, Sarina G Kant ${ }^{21}$, Erica H Gerkes ${ }^{3}$, Helen V Firth ${ }^{22}$, Katrin Ounap ${ }^{23}$, \\ Lynne M Bird ${ }^{24}$, Diane Masser-Frye ${ }^{24}$, Jennifer R Friedman ${ }^{24}$, Modupe A Sokunbi ${ }^{25}$, Abhijit Dixit ${ }^{26}$, \\ Miranda Splitt ${ }^{27}$, DDD Study, Mary K Kukolich ${ }^{28}$, Julie McGaughran ${ }^{29}$, Bradley P Coe ${ }^{30}$, Jesús Flórez ${ }^{31}$, \\ Nael Nadif Kasri ${ }^{1}$, Han G Brunner ${ }^{1,32}$, Elizabeth M Thompson ${ }^{33}$, Jozef Gecz ${ }^{34}$, Corrado Romano ${ }^{19}$, \\ Evan E Eichler ${ }^{30,35}$ and Bert BA de Vries ${ }^{1}$
}

The Koolen-de Vries syndrome (KdVS; OMIM \#610443), also known as the 17q21.31 microdeletion syndrome, is a clinically heterogeneous disorder characterised by (neonatal) hypotonia, developmental delay, moderate intellectual disability, and characteristic facial dysmorphism. Expressive language development is particularly impaired compared with receptive language or motor skills. Other frequently reported features include social and friendly behaviour, epilepsy, musculoskeletal anomalies, congenital heart defects, urogenital malformations, and ectodermal anomalies. The syndrome is caused by a truncating variant in the KAT8 regulatory NSL complex unit 1 (KANSL1) gene or by a 17q21.31 microdeletion encompassing KANSL1. Herein we describe a novel cohort of 45 individuals with KdVS of whom 33 have a 17q21.31 microdeletion and 12 a single-nucleotide variant (SNV) in KANSL1 (19 males, 26 females; age range 7 months to 50 years). We provide guidance about the potential pitfalls in the laboratory testing and emphasise the challenges of KANSL1 variant calling and DNA copy number analysis in the complex 17q21.31 region. Moreover, we present detailed phenotypic information, including neuropsychological features, that contribute to the broad phenotypic spectrum of the syndrome. Comparison of the phenotype of both the microdeletion and SNV patients does not show differences of clinical importance, stressing that haploinsufficiency of KANSL1 is sufficient to cause the full KdVS phenotype.

European Journal of Human Genetics (2016) 24, 652-659; doi:10.1038/ejhg.2015.178; published online 26 August 2015

\section{INTRODUCTION}

The Koolen-de Vries syndrome (KdVS) (OMIM \#610443), also known as the $17 \mathrm{q} 21.31$ microdeletion syndrome, is a multisystem condition characterised by developmental delay, intellectual disability (ID), hypotonia, epilepsy, characteristic facial features, and congenital malformations in multiple organ systems. ${ }^{1-3}$ The syndrome can be

${ }^{1}$ Department of Human Genetics, Radboud Institute for Molecular Life Sciences and Donders Institute for Brain, Cognition and Behaviour, Radboud University Medical Center, Nijmegen, The Netherlands; ${ }^{2}$ Department of Clinical Genetics, VU University Medical Center, Amsterdam, The Netherlands; ${ }^{3}$ Department of Genetics, University of Groningen, University Medical Center Groningen, Groningen, The Netherlands; ${ }^{4}$ Department of Laboratories, Seattle Children's Hospital, Seattle, WA, USA; ${ }^{5}$ Unidade de Genética Médica, Centro de Genética Médica Dr Jacinto Magalhães, Centro Hospitalar do Porto, Porto, Portugal; ${ }^{6}$ Institut für Humangenetik, University of Luebeck, Luebeck, Germany; ${ }^{7}$ Genetics Department, Children's Hospitals and Clinics of Minnesota, Minneapolis, MN, USA; ${ }^{8}$ Department of Human Genetics, University of Chicago, Chicago, IL, USA; ${ }^{9}$ Department of Pediatrics, University of Mississippi Medical Center, Jackson, MS, USA; ${ }^{10}$ St Luke's Pediatric Associates, Duluth, MN, USA; ${ }^{11}$ Department of Pediatrics and Medicine, Columbia University, New York, NY, USA; ${ }^{12}$ Counties Manukau District Health Board, South Auckland, New Zealand; ${ }^{13}$ Epilepsy Center, St Ivan Rilski University Hospital, Sofia, Bulgaria; ${ }^{14}$ Department of Pediatrics, Medical Genetics University of Catania, Catania, Italy; ${ }^{15}$ Clinical Genetics, Yorkshire Regional Genetics Service, Leeds, UK; ${ }^{16}$ Department of genetics, Yale University School of Medicine, New Haven, CT, USA; ${ }^{17}$ Division of Human Genetics, Cincinnati Children's Hospital Medical Center, Cincinnati, OH, USA; ${ }^{18}$ Department of Medical Genetics, University of Antwerp, Antwerp, Belgium; ${ }^{19}$ Pediatrics and Medical Genetics, I.R.C.C.S. Associazione Oasi Maria Santissima, Troina, Italy; ${ }^{20}$ Department of Clinical Genetics, Erasmus MC, Sophia Children's Hospital, Rotterdam, The Netherlands; ${ }^{21}$ Department of Clinical Genetics, Leiden University Medical Center, Leiden, The Netherlands; ${ }^{22}$ Department of Medical Genetics, Cambridge University Addenbrooke's Hospital, Cambridge, UK; ${ }^{23}$ Department of Genetics, United Laboratories, Tartu University Hospital, Tartu, Estonia; ${ }^{24}$ Departments of Neurosciences and Pediatrics, University of California San Diego, and Divisions of Neurology and Genetics, Rady Children's Hospital San Diego, San Diego, CA, USA; ${ }^{25}$ Nacogdoches Pediatrics, Nacogdoches, TX, USA; ${ }^{26}$ Clinical Genetics, Nottingham City Hospital, Nottingham, UK; ${ }^{27}$ Northern Genetic Service, Institute of Genetic Medicine, Newcastle upon Tyne, UK; ${ }^{28}$ Clinical Genetics, Cook Children's Hospital, Fort Worth, TX, USA; ${ }^{29}$ Genetic Health Queensland, Royal Brisbane and Women's Hospital and School of Medicine, University of Queensland, Brisbane, Queensland, Australia; ${ }^{30}$ Department of Genome Sciences, University of Washington School of Medicine, Seattle, WA, USA; ${ }^{31}$ Department of Physiology and Pharmacology, University of Cantabria, Cantabria, Spain; ${ }^{32}$ Department of Clinical Genetics, Maastricht University Medical Center, Maastricht, The Netherlands; ${ }^{33}$ South Australian Clinical Genetics Service, Women's and Children's Hospital; and Department of Paediatrics, University of Adelaide, Adelaide, South Australia, Australia; ${ }^{34}$ School of Paediatrics and Reproductive Health and Robinson Research Institute, The University of Adelaide at the Women's and Children's Hospital, North Adelaide, South Australia, Australia; ${ }^{35}$ Howard Hughes Medical Institute, University of Washington, Seattle, WA, USA

*Correspondence: Dr DA Koolen, Department of Human Genetics, Radboud University Medical Center, P0 Box 9101 , Nijmegen 6500 HB, The Netherlands. Tel: +0031 24 13946; Fax: +0031 24 3668753; E-mail: david.koolen@radboudumc.nl

Received 28 April 2015; revised 21 June 2015; accepted 24 June 2015; published online 26 August 2015 
either caused by a microdeletion in chromosomal region $17 \mathrm{q} 21.31$ or by a truncating variant in the KAT8 regulatory NSL complex unit 1 (KANSL1) gene (NG_032784.1). ${ }^{4,5}$

In 2012, pathogenic single-nucleotide variants (SNVs) in KANSL1 in four independent individuals with the core clinical features of the 17q21.31 microdeletion syndrome were identified, showing that haploinsufficiency of KANSL1 is sufficient to cause the full $17 \mathrm{q} 21.31$ deletion phenotype. ${ }^{4,5}$ KANSL1 is known to be part of the non-specific lethal (NSL) complex, which regulates global transcription by histone modification. ${ }^{6}$ The complex consists of several proteins, including KANSL1, KANSL2, KANSL3, WDR5, MCRS1, and PHF20, and the histone acetyltransferase KAT8. ${ }^{7-9}$

The recurrent 17q21.31 deletion (hg19 chr17:g.43700000_ 44300000) contains several genes, including CRHR1 (OMIM ${ }^{\star} 122561$ ), STH (OMIM *607067), MAPT (OMIM $\left.{ }^{\star} 157140\right)$, and KANSL1 (OMIM ${ }^{\star} 612452$ ), and is flanked by extensive low copy repeats (LCRs) or segmental duplications. The locus is genomically complex with multiple structurally diverse haplotypes segregating in the human population. ${ }^{10,11}$ Most fall into two genetically distinct haplotypes: the $\mathrm{H} 1$ haplotype, which is most common, and the inverted H2 haplotype, which is present at a frequency of $\sim 20 \%$ in European chromosomes. ${ }^{12}$ We previously showed that there is a $\mathrm{H} 2$ predisposition to the microdeletion, ${ }^{13}$ which can be explained by the fact that the most prevalent $\mathrm{H} 2$ haplotype contains homologous segmental duplications in direct orientation flanking the diseasecritical region, whereas no such sequence is observed in the $\mathrm{H} 1$ haplotype. ${ }^{10,11,14}$ Itsara et $a l^{15}$ showed that the $17 \mathrm{q} 21.31$ deletion breakpoints reside within the flanking homologous sequences and that the deletion can be mediated by intrachromosomal non-allelic homologous recombination involving the $\mathrm{H} 2$-specific LCRs in direct orientation or by an interchromosomal unequal crossover between the $\mathrm{H} 1$ and $\mathrm{H} 2$ haplotypes.

So far, clinical data on 81 individuals with KdVS have been reported in the medical literature, including only four individuals with a KANSL1 SNV. ${ }^{1-5,13,16-32}$ The rarity of identified patients with a pathogenic SNV in KANSL1 hampers the study of genotype-phenotype correlations. Moreover, despite the relatively high number of cases with a deletion, the full clinical spectrum of the syndrome is still unknown and better natural history data are needed.

We herein describe a novel cohort of 45 individuals with KdVS of whom 33 have a chromosome 17q21.31 deletion and 12 a SNV in KANSL1. We correlate our data to that in the medical literature and provide practical information on the molecular and clinical interpretation of copy number variation and single-nucleotide variation in the complex 17q21.31 region.

\section{SUBJECTS AND METHODS}

Thirty-three individuals with a 17q21.31 deletion (14 males, 19 females, mean age 11.9 years, range 7 months to 50.1 years) and 12 individuals with a pathogenic intragenic KANSL1 SNV (5 male, 7 female, mean age 11.8 years, range $2.9-46$ years) were included in the study. The deletions were detected by whole-genome array analysis with different platforms (Affymetrix, Santa Clara, CA, USA; Agilent, Santa Clara, CA, USA; Illumina, San Diego, CA, USA). In six cases, the KANSL1 variant was identified using whole-exome sequencing (cases $35,38,39,41,42,44)$, two of which originated from the Deciphering Developmental Disorders (DDD) project (www.ddduk.org/). ${ }^{33}$ One variant (case 40) was identified using a molecular inversion probes (MIP) assay. ${ }^{34}$ Other patients underwent direct sequencing of KANSL1 owing to clinical evidence of KdVS phenotype. The 17q21.31 deletions and KANSL1 sequence variants were submitted to the Leiden Open Variation Database (http:// databases.lovd.nl/shared/variants and http://www.lovd.nl/KANSL1). ${ }^{35}$
A subset $(n=9)$ included in this study were diagnosed at the Department of Human Genetics, Radboud University Medical Center, Nijmegen, The Netherlands. The remaining participants were from clinicians at collaborating centers. The clinical data on 42 patients have not been reported previously; cases 25,34 , and 43 were previously published. ${ }^{4,31}$ A total of 12 individuals underwent detailed clinical examination by a clinical geneticist of the Department of Human Genetics in Nijmegen. For the remaining cases, clinical information was collected from other clinicians using a standardised checklist (Supplementary Table S1). A search of the medical literature identified 23 articles describing the clinical features of 81 patients with KdVS. ${ }^{1-5,13,16-32}$

\section{RESULTS}

\section{Genotype}

All deletions in our cohort of 33 individuals include CRHR1, SPPL2C, $M A P T, S T H$, and at least the $3^{\prime}$ end of the KANSL1 gene (Supplementary Figure S1). The deletion size, as determined by various array platforms, varied between 378 and $645 \mathrm{~kb}$. The 12 pathogenic KANSL1 variants were scattered throughout the entire gene (NM_001193466.1), although 5 variants were located in exon 2. The pathogenic variants are shown in Figure 1 and summarised in Table 1. All variants predicted protein truncation, including one nonsense variant, eight frame shift variants, and three canonical splice site variants. The heterozygous variant within exon 15, p.(Leu1042Argfs ${ }^{\star} 71$ ) (case 44), is predicted to result in a shift in the reading frame, reaching a stop codon after 71 codons, altering the C-terminal end of the KANSL1 protein. Notably, the c.1652+1G > A splice site variant was reported in two unrelated individuals (cases 35 and 43).

\section{Genetic testing}

Genetic testing of the $17 q 21.31$ genomic region can be challenging. The mapping and interpretation of the deletion breakpoints are confounded by the structural complexity and genomic variation of the $17 \mathrm{q} 21.31$ locus. The resolution of the different array platforms, for example, is limited because of the low probe coverage of the segments that are rich in LCRs. Moreover, the region consists of two (benign) copy-number polymorphic (CNP) segmental duplications, including a short duplication (CNP155 or $\alpha$ polymorphism duplication) and a long duplication (CNP205 or $\beta$ polymorphism duplication) corresponding to the promoter and first exons of KANSL1 (Supplementary Figure S1 and Supplementary Figure S2a). ${ }^{10,11}$ These CNPs are associated with the $\mathrm{H} 2$ and $\mathrm{H} 1$ haplotypes, respectively, and are significantly enriched in European/Mediterranean populations where frequencies can reach as high as $60 \% .{ }^{10,11}$ These common copy number variants (CNVs) are listed in large numbers in the various databases as inherited and/or benign (ISCA benign variants (http:// dbsearch.clinicalgenome.org/search/); Decipher (http://decipher.sanger.ac.uk); Database of Genomic Variants (http://dgv.tcag.ca/) and can give rise to 'artefacts' in genetic testing. Genotyping the two CNPs in the 1000 Genomes ${ }^{36}$ phase 3 data identified frequent duplication of these regions with no observed deletions (Supplementary Figure S2b). Because of the lack of deletions, we examined the raw array ratio data from a representative set of 8730 controls. ${ }^{34}$ In both CNP clusters, the high frequency of variants had shifted the central cluster towards lower ratios (Supplementary Figure S2c). This results in both false calling of deletions in diploid state and undercalling of duplications. Thus array data representing a deletion in the duplicated portion of KANSL1 should be viewed with caution unless a carefully selected control set (in the case of SNP platforms) or well-characterised control (for array CGH) was used. These data show that especially distal events in 

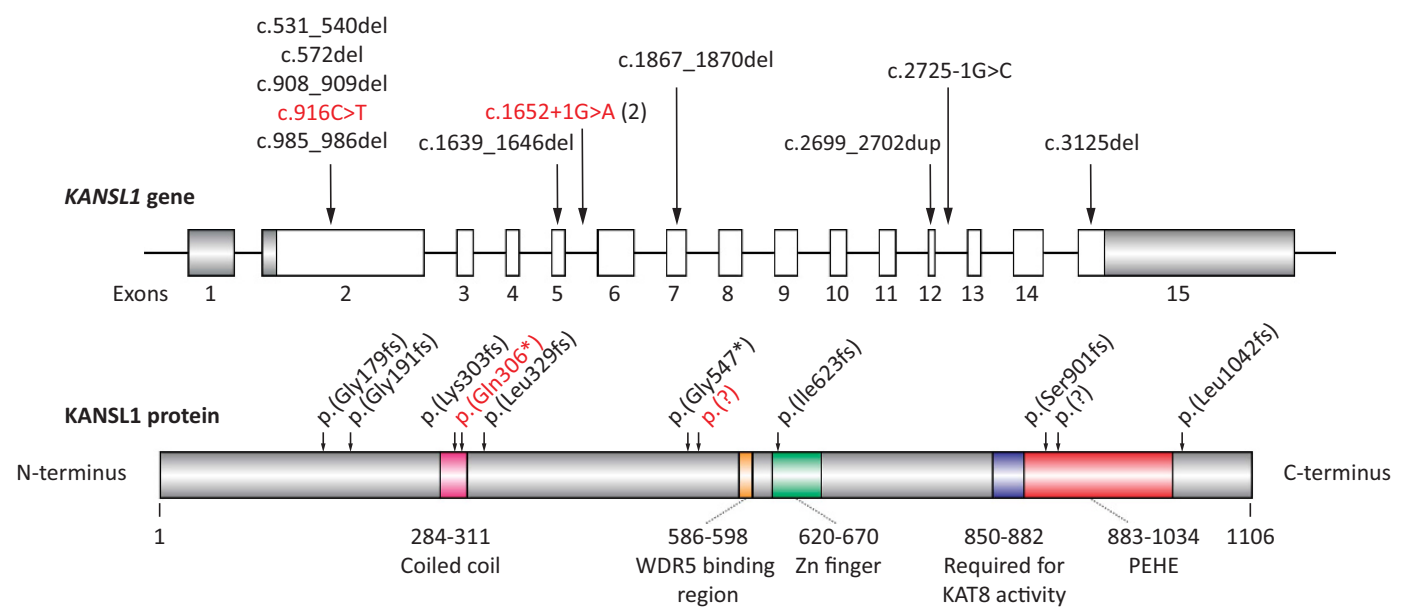

Figure 1 Structure of the KANSL1 gene and protein and distribution of the SNVs. (a) Gene structure of KANSL1 (NG_032784.1). White boxes indicate exons, connecting lines indicate introns (not drawn on scale), and grey boxes represent untranslated regions (UTRs). SNVs are annotated at the cDNA followed by the number of cases (if $>1$ ). (b) Linear protein structure of KANSL1 indicating the different domains and motifs. The KANSL1 protein is mainly unstructured, except for a coiled-coil domain (CC), a possible Zinc finger domain (ZN), a WDR5 interacting motif (WIN), and a PEHE domain. ${ }^{6}$ Amino-acid changes are shown above the protein with arrows indicating their position. The two variants that were previously described in the medical literature are shown in red.

Table 1 Variants in KANSL1 (NM_001193466.1) and their predicted effects at the protein level

\begin{tabular}{|c|c|c|c|c|c|c|}
\hline Case & Genomic position & Mutation (nt) & Change (aa) & Exon & Mutation type & De novo \\
\hline \#34 & chr17:g.44248594G >A & c. $916 \mathrm{C}>\mathrm{T}$ & p. $\left(\mathrm{G} \ln 306^{*}\right)$ & Exon 2 & Nonsense & Yes \\
\hline \#35 & chr17:g.44144914C > T & c. $1652+1 G>A$ & p.(?) & Intron 5 & Splice site & Yes \\
\hline \#36 & chr17:g.44248524_44248525del & c.985_986del & p.(Leu329Glufs*22) & Exon 2 & Frameshift & Yes \\
\hline \#37 & chr17:g.44128049_44128052del & c.1867_1870del & p.(Ile623Alafs*6) & Exon 7 & Frameshift & NA \\
\hline \#38 & chr17:g.44248970_44248979del & c.531_540del & p.(Gly179Leufs*20) & Exon 2 & Frameshift & Yes \\
\hline \#39 & chr17:g.44110559C > G & c. $2725-1 G>C$ & p.(?) & Intron 12 & Splice site & Yes \\
\hline \#40 & chr17:g.44144921_44144928del & c.1639_1646del & p.(Gly547*) & Exon 5 & Frameshift & NA \\
\hline \#41 & chr17:g.44248938del & c.572del & p.(Gly191Valfs*11) & Exon 2 & Frameshift & Yes \\
\hline \#42 & chr17:g.44110791_44110794dup & c.2699_2702dup & p.(Ser901Argfs*4) & Exon 12 & Frameshift & Yes \\
\hline \#43 & chr17:g.44144914C > T & c. $1652+1 \mathrm{G}>\mathrm{A}$ & p.(?) & Intron 5 & Splice site & Yes \\
\hline \#44 & chr17:g.44109035del & c.3125del & p.(Leu1042Argfs*71) & Exon 15 & Frameshift & Yes \\
\hline \#45 & chr17:g.44248601_44248602del & c.908_909del & p.(Lys303Thrfs*11) & Exon 2 & Frameshift & NA \\
\hline
\end{tabular}

Abbreviations: aa, amino acid; nt, nucleotide; NA, DNA of parent(s) not available. Summary of all sequence variants found in KANSL1 in the individuals reported herein. Genomic, cDNA, and protein positions are given as well as the affected exons. All genomic positions are based on the GRCh37/hg19 sequence; KANSL1 genomic reference sequence NG_032784.1; and transcript protein positions are given as well as the
reference sequence NM 001193466.1.

KANSL1 need careful clinical examination in order to confirm the diagnosis of KdVS.

On SNP-based microarray array platforms, the deep coalescence (2.3 million years) and the sequence divergence of the $\mathrm{H} 2$ inversion haplotype can also hinder proper local genotype calling often visible as disturbances in the KANSL1 region in the B-allele frequency/allele peaks plots. We have seen that different $\mathrm{H} 1 / \mathrm{H} 2$ genotypes give rise to a specific and recognisable pattern on various array platforms depending on the parental duplication structures (personal communication $\mathrm{T}$ Dijkhuizen; Nijmegen examples of CytoscanHD platform provided in Supplementary Figure S3).

In addition to difficulties with the standard $\mathrm{CNV}$ analysis, the $\mathrm{H} 2$ haplotype and the CNP clusters encompassing exons 1-3 of KANSL1 contribute to difficulties in SNV calling such as loss-of-function variant 'artefacts' in the KANSL1 gene. As a result, in a number of variant databases, we have observed loss-of-function variants reported in non-affected individuals (the Exome Aggregation Consortium, http://exac.broadinstitute.org/; NHLBI GO Exome Sequencing Project,
http://evs.gs.washington.edu/EVS/) corresponding specifically to the duplicated portion. This means that the detection of a truncating variant in exons 1-3 of the KANSL1 gene is not sufficient to make a diagnosis of KdVS. In these cases, next to a compatible clinical phenotype, variant analysis of the parental samples is of the utmost importance to make sure that the possibly pathogenic variant has originated 'de novo'. The latter is illustrated by a nonsense variant in exon 2 of KANSL1 that was identified in the DNA of a man with ID using the MIP approach. ${ }^{34}$ Other than ID, the clinical presentation did not suggest KdVS. We subsequently tested the DNA of both parents and showed that the unaffected mother also carried the KANSL1 variant.

\section{Clinical phenotype}

In total, we collected clinical information on 33 patients with a $17 q 21.31$ deletion and 12 with a pathogenic KANSL1 variant. All patients were scored using a clinical checklist, allowing us to study genotype-phenotype correlations. The KANSL1 variants were scattered 
throughout the gene (Figure 1 and Table 1) and no genotypephenotype correlations were apparent in the cohort of patients with a pathogenic variant.

Table 2 Growth parameters and the key clinical features of KdVS

\begin{tabular}{|c|c|c|c|c|c|c|}
\hline \multirow[b]{2}{*}{ Clinical features } & \multicolumn{2}{|c|}{$\begin{array}{l}17 q 21.31 \\
\text { deletion } \\
(n=33)\end{array}$} & \multicolumn{2}{|c|}{$\begin{array}{l}\text { KANSL1 } \\
\text { variant } \\
(n=12)\end{array}$} & \multicolumn{2}{|c|}{$\begin{array}{l}\text { KdVS } \\
\text { total } \\
(n=45)\end{array}$} \\
\hline & $n$ & $\%$ & $n$ & $\%$ & $n$ & $\%$ \\
\hline \multicolumn{7}{|l|}{ Growth parameters } \\
\hline Low birth weight & 7/23 & 30.4 & $2 / 12$ & 16.7 & 9/35 & 25.7 \\
\hline Postnatal short stature & $10 / 24$ & 41.7 & $2 / 10$ & 20.0 & $12 / 34$ & 35.3 \\
\hline Microcephaly & $1 / 25$ & 4.0 & $0 / 10$ & 0.0 & $1 / 35$ & 2.9 \\
\hline Macrocephaly & $3 / 25$ & 12.0 & $2 / 10$ & 20 & $5 / 35$ & 14.3 \\
\hline \multicolumn{7}{|l|}{ Neurological features } \\
\hline Hypotonia & $28 / 32$ & 87.5 & $10 / 12$ & 83.3 & $38 / 44$ & 86.4 \\
\hline Feeding problems & $19 / 25$ & 76.0 & $8 / 12$ & 66.7 & $27 / 37$ & 73.0 \\
\hline DD/intellectual disability & $33 / 33$ & 100.0 & $12 / 12$ & 100.0 & $45 / 45$ & 100.0 \\
\hline Mild & $13 / 30$ & 43.3 & $4 / 11$ & 36.3 & $17 / 41$ & 41.5 \\
\hline Moderate & $11 / 30$ & 36.7 & $4 / 11$ & 36.3 & $15 / 41$ & 36.6 \\
\hline Severe & $6 / 30$ & 20.0 & $3 / 11$ & 27.2 & $9 / 41$ & 22.0 \\
\hline Motor delay & $25 / 25$ & 100.0 & $11 / 12$ & 91.7 & $36 / 37$ & 97.3 \\
\hline Speech delay & $24 / 24$ & 100.0 & $12 / 12$ & 100.0 & $36 / 36$ & 100.0 \\
\hline Seizures/EEG anomalies & $15 / 33$ & 45.5 & $7 / 12$ & 58.3 & $22 / 45$ & 48.9 \\
\hline Friendly/amiable affect & $26 / 30$ & 88.7 & $11 / 12$ & 91.7 & $37 / 42$ & 88.7 \\
\hline Structural CNS anomalies & $15 / 26$ & 57.7 & $3 / 8$ & 37.5 & $18 / 34$ & 52.9 \\
\hline Enlarged ventricles & $10 / 27$ & 37.0 & $3 / 9$ & 33.3 & $13 / 36$ & 36.1 \\
\hline Neuropsychological disorders & $18 / 32$ & 56.3 & $7 / 12$ & 58.3 & $25 / 44$ & 56.8 \\
\hline \multicolumn{7}{|l|}{ Dysmorphic features } \\
\hline Long face & $23 / 32$ & 71.9 & $10 / 12$ & 83.3 & $33 / 44$ & 75.0 \\
\hline Upslanting palpebral fissures & $16 / 33$ & 48.5 & $8 / 11$ & 72.7 & $24 / 44$ & 54.5 \\
\hline Narrow/short palpebral fissures & $14 / 24$ & 58.3 & $5 / 10$ & 50.0 & $19 / 34$ & 55.0 \\
\hline Ptosis & $12 / 33$ & 36.4 & $2 / 11$ & 18.1 & $14 / 44$ & 31.8 \\
\hline Epicanthal folds & $18 / 33$ & 54.5 & $5 / 11$ & 45.5 & $23 / 44$ & 52.3 \\
\hline Tubular or pear-shaped nose & $27 / 32$ & 84.4 & $7 / 11$ & 63.6 & $34 / 43$ & 81.0 \\
\hline Bulbous nasal tip & $29 / 32$ & 90.6 & 9/11 & 81.8 & $38 / 43$ & 88.3 \\
\hline Everted lower lip & $15 / 24$ & 66.7 & 9/11 & 81.8 & $25 / 35$ & 71.4 \\
\hline Large/prominent ears & $14 / 32$ & 43.8 & $0 / 11$ & 0.0 & $14 / 43$ & 32.6 \\
\hline Musculoskeletal anomalies & $25 / 32$ & 78.1 & $8 / 11$ & 72.7 & $33 / 43$ & 76.7 \\
\hline Tracheo/laryngomalacia & $5 / 33$ & 15.2 & $4 / 11$ & 36.3 & $9 / 44$ & 20.9 \\
\hline Scoliosis/kyphosis & $13 / 33$ & 39.4 & $4 / 12$ & 33.3 & $17 / 45$ & 37.8 \\
\hline Joint hyperlaxity & $17 / 30$ & 56.7 & $7 / 11$ & 63.6 & $24 / 41$ & 58.5 \\
\hline Positional deformity feet & $12 / 31$ & 38.7 & $8 / 11$ & 72.7 & $20 / 42$ & 47.6 \\
\hline Visual and hearing impairments & $16 / 29$ & 55.2 & $8 / 9$ & 72.7 & $24 / 38$ & 63.2 \\
\hline Hypermetropia & $10 / 31$ & 32.3 & $6 / 11$ & 54.5 & $16 / 42$ & 38.0 \\
\hline Strabismus & $12 / 33$ & 36.4 & $6 / 11$ & 54.5 & $18 / 44$ & 40.9 \\
\hline Hearing impairment & 9/28 & 32.1 & $1 / 12$ & 8.3 & $10 / 40$ & 25.0 \\
\hline Heart defects & $11 / 33$ & 33.3 & $6 / 11$ & 54.5 & $17 / 44$ & 38.6 \\
\hline Renal and urogenital anomalies & $14 / 32$ & 43.8 & $5 / 10$ & 50.0 & $19 / 42$ & 45.2 \\
\hline Cryptorchidism & $7 / 14$ & 50.0 & $3 / 5$ & 60.0 & $10 / 19$ & 52.6 \\
\hline Ectodermal abnormalities & $21 / 32$ & 65.6 & $7 / 10$ & 70.0 & $28 / 42$ & 66.7 \\
\hline Multiple moles & $5 / 33$ & 15.2 & $3 / 11$ & 27.3 & $8 / 44$ & 18.2 \\
\hline
\end{tabular}

Next all individuals were separated according to genotypes (17q21.31 deletion versus pathogenic KANSL1 variant) (Table 2). Comparison of the phenotypes of both groups of patients did not show differences of clinical importance, stressing the previous conclusion that haploinsufficiency of KANSL1 is sufficient to cause the core phenotype of KdVS. ${ }^{4,5}$ We did not observe any significant differences between the two groups, except for large and/or prominent ears in the deletion group (44\%). This feature was not reported in the group with SNVs, giving a significant uncorrected $P$-value of 0.017 (Fisher's Exact Test). Otherwise, there were striking facial similarities between both the groups (Figure 2 and Figure 3).

The individual clinical signs of our cohort of 45 patients are shown in Supplementary Table S1. Clinical features of KdVS were apparent in all individuals with the deletion or a pathogenic KANSL1 variant, although the extent and severity of clinical findings varied among individuals. The growth parameters and the features reported most frequently in KdVS (ie, in $\geq 30 \%$ of the $17 q 21.31$ deletion group, the KANSL1 SNV group, or both groups) are shown in Table 2. Clinical photographs of the patients are shown in Figures 2 and 3. A summary of the main systems involved is given below.

\section{Growth parameters}

In the current cohort of 45 individuals with KdVS, intrauterine growth retardation (IUGR) was recorded in 26\%. This is similar to numbers in the medical literature (18-29\%)..$^{13,18,22}$ Postnatal growth retardation is present in $35 \%$, which is also similar to the other larger cohorts of patients (18-36\%). ${ }^{13,22}$ Short stature, if present, was proportionate. In the current cohort, case 17 with a 17q21.31 deletion and case 42 with a KANSL1 variant had (partial) growth hormone deficiency, which has been reported previously. ${ }^{13,19}$

\section{Neurological features}

All individuals with KdVS in our cohort have developmental delay and ID. The level of ID is mild in $42 \%$, moderate in $37 \%$ and severe in $22 \%$. In case 36 , a girl with a KANSL1 variant and West syndrome, the level of delay was, remarkably, only mild (IQ 73). Borderline IQ was also described in two boys with a 17q21.31 deletion who finished secondary school with educational support. ${ }^{20}$ However, in general the level of psychomotor delay is moderate and expressive language development seems to be particularly affected compared with receptive language or motor skills. In our cohort, 4 out of 35 informative cases (ie, age $\geq 5$ years) were non-verbal ( $11 \%)$.

Neonatal hypotonia is a key feature of KdVS, reported in $86 \%$ of all cases. It may result in severe feeding difficulties and hospitalisation and/or nasogastric tube feeding in the neonatal period. Hypotonia is present mainly during infancy but can continue into adulthood. Also, feeding difficulties and oral motor dyspraxia, including drooling, gastro-oesophageal reflux, and aspiration pneumonia (case 12), can give rise to problems later in life. In case 42, severe drooling (sialorroea) required resection of the submandibular salivary glands.

Seizures were present in $49 \%$ of all cases and were generally well controlled with antiepileptic medications. In the medical literature seizures are described in 36-54\%. ${ }^{13,18,22}$ Wray $^{25}$ described a girl with infantile spasms presenting at 5 months of age and suggested that the deletion of CRHR1 may predispose to having seizures, especially to infantile spasms. However, in the current cohort infantile spasms were also reported in case 41 with a KANSL1 variant.

Structural anomalies of the central nervous system were present in $53 \%$ of our cohort and mainly included corpus callosum hypoplasia/aplasia, enlarged ventricles, hydrocephalus, and heterotopias. These features have also been reported previously in addition to 

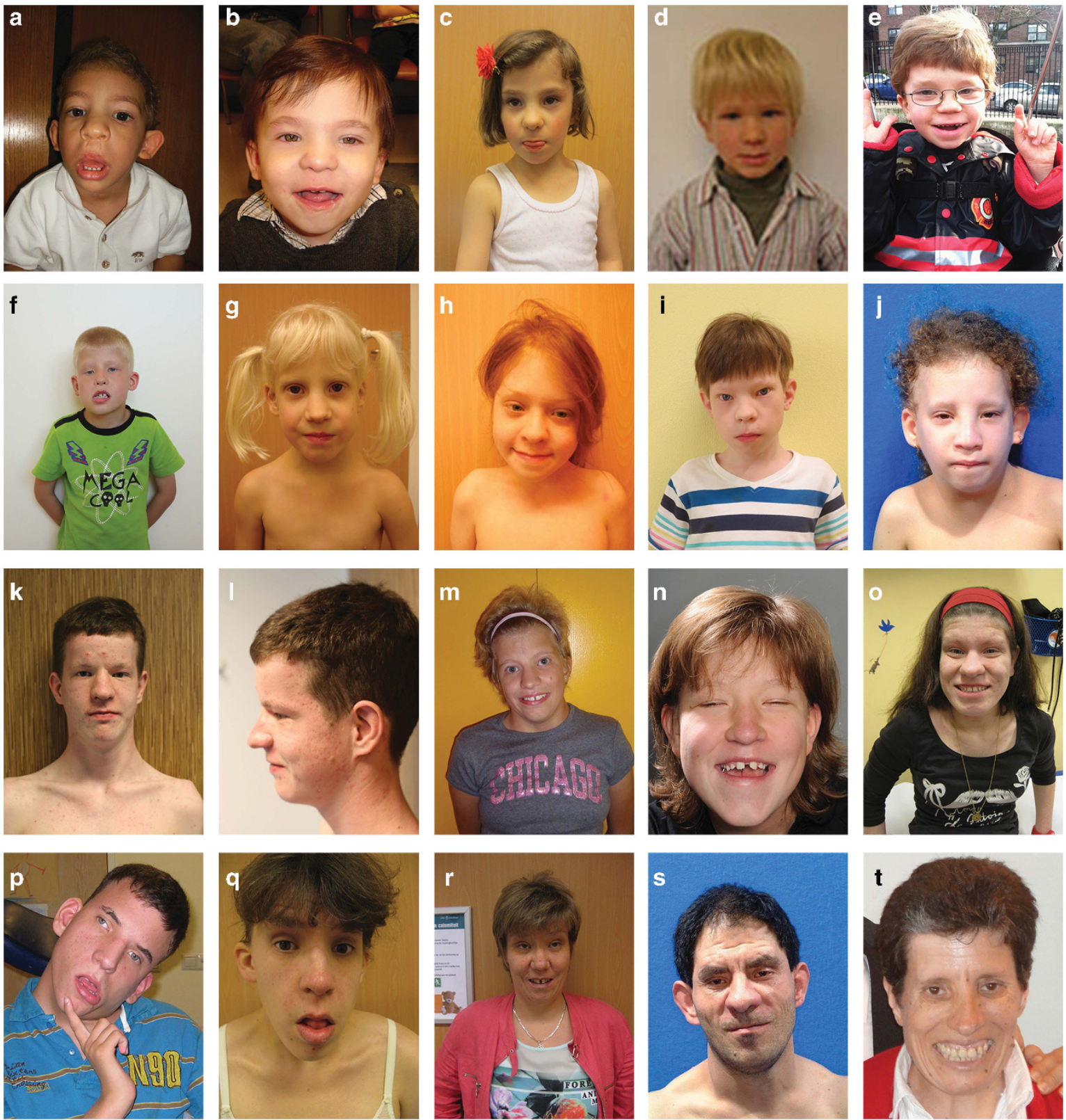

Figure 2 Clinical photographs of individuals with KdVS due to a 17q21.31 deletion. Facial photographs of (a) case 5; (b) case 7; (c) case 10; (d) case 11; (e) case 12; (f) case 14; (g) case 16; (h) case 17; (i) case 18; (j) case 22; ( $\mathbf{k})$ case 24 front view; (I) case 24 side view; (m) case 25; (n) case 26; (o) case 28; (p) case 29; (q) case 30; (r) case 31; (s) case 32; and (t) case 33. A written informed consent was obtained for publication of clinical photographs in the medical literature.

communicating hydrocephalus, periventricular white matter changes, ${ }^{13,22,29}$ and partial pituitary stalk interruption syndrome. ${ }^{19}$ Case 35 presented with a Chiari type 1 malformation, which has also been described by Terrone $e t a l,{ }^{23}$ co existing with a mild anomaly of the medulla oblongata. Three cases in our cohort had spinal cord anomalies, including tethered cord (case 13), hydromyelia of the thoracic cord (case 38), and painful disabling dural ectasia and cysts (case 45). Interestingly, Tan et $a l^{22}$ also described an individual with scoliosis, dural ectasia and a Tarlov cyst.

\section{Neuropsychological disorders}

In general, individuals with KdVS are sociable and have an amiable affect. ${ }^{13,18,22}$ This is also reported in our cohort in $89 \%$ of all cases.
However, behavioural problems such as hyperactive behaviour, anxiety, and stammering have also been reported. ${ }^{13,18,20,22,30}$ In our cohort, we observed behavioural problems in $57 \%$ of cases. These problems included autism/autistic traits, ADHD/attention deficit/ hyperactivity, shyness, anxiety/phobias, impulsive and stereotypic behaviour, psychosis, and depression. Case 41, a 10-year-old girl with a KANSL1 SNV, for example, had a disturbed sleep pattern, dependence on routines, and self-stimulating behaviours (eye poking and chewing on fingers). Case 44, a 20-year-old man with a KANSL1 $\mathrm{SNV}$, had mild perseverative behaviour and anxiety in early childhood, with worsening in early adolescence. He had anxiety and fluctuating aggressive/agitated behaviours and developed severe perseverative/ obsessive compulsive behaviours in late adolescence that interfered 

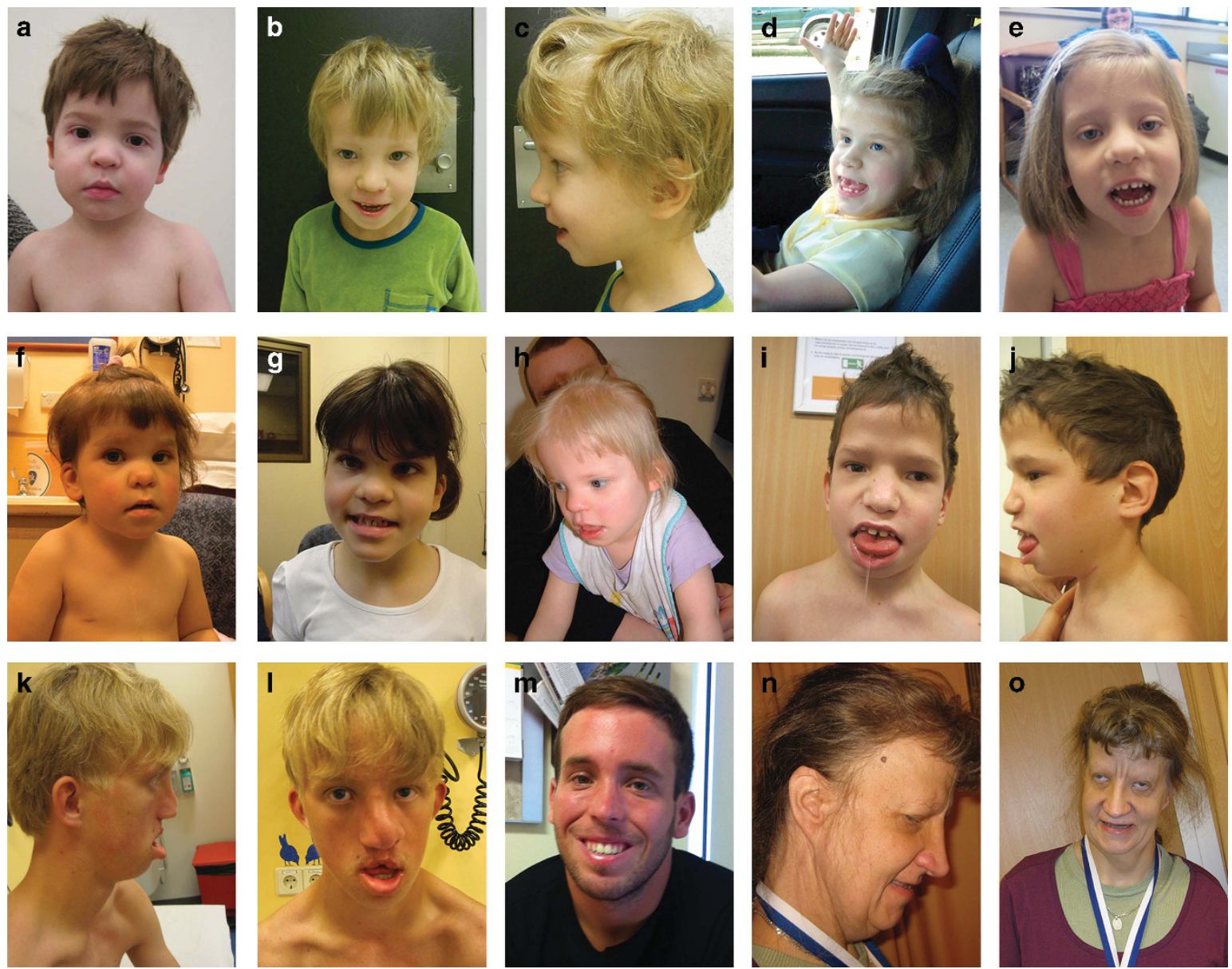

Figure 3 Clinical photographs of individuals with KdVS due to a pathogenic KANSL1 variant. Facial photographs of (a) case 35; (b) case 36 front view; (c) case 36 side view; (d) case 38; (e) case 39; (f) case 40 age 2 years; (g) case 40 age 7 years; (h) case 41 (i) case 42 front view; (j) case 42 side view; (k) case 43 side view; (I) case 43 front view; (m) case 44; (n) case 45 side view; and (o) case 45 front view. A written informed consent was obtained for publication of clinical photographs in the medical literature.

with activities of daily living. Some regression was also reported in case 33 , a 50-year-old woman with a 17q21.31 deletion. While at one point she wrote clearly and precisely, over the years she lost that ability. She had a tendency towards obsessive thoughts that have persisted her whole life. In addition, she developed situational depression on two occasions. A more detailed description of her is provided in the Supplementary Note.

\section{Facial dysmorphism}

The most prominent facial dysmorphisms present in our series of 45 cases are the long face, upslanting palpebral fissures, narrow/short palpebral fissures, ptosis, epicanthal folds, tubular- or pear-shaped nose $(81 \%)$ with bulbous nasal tip (88\%), everted lower lip, and large prominent ears (Figure 2 and Figure 3 ). A high and narrow palate has been described in eight cases as another frequent feature. The facial features are similar between the $17 \mathrm{q} 21.31$ deletion group (Figure 2) and the KANSL1 SNV group (Figure 3). With age, there is elongation and coarsening of the face. An abnormally shaped skull is reported in 9 cases, including dolichocephaly (cases 15, 25, 35), metopic ridge (case 15), bitemporal narrowing (cases 22, 23, 24), trigonocephaly (case 24), brachycephaly (case 29), turricephaly/scaphocephaly (case 32), and frontal bossing (case 41). Craniosynostosis as well as minor alterations in skull shape have been described previously. ${ }^{13,18,21,22}$
Other nonfacial dysmorphic features that have been reported in the medical literature include fetal finger pads, ${ }^{22}$ slender lower limbs, ${ }^{13}$ and postaxial polydactyly of fingers and toes. ${ }^{24,32}$

\section{Musculoskeletal anomalies}

Musculoskeletal anomalies were present in $77 \%$ of our cases and included tracheo/laryngomalacia, pectus excavatum or carinatum, scoliosis/kyphosis, hip dislocation/dysplasia, joint hypermobility, and positional deformities of the feet. Tracheo/laryngomalacia (21\%) can occasionally result in severe respiratory problems (case 38 needed supraglottoplasty twice). Joint hypermobility mainly involved the fingers and hands, whereas the positional deformities of the feet can be severe and included pes planus but also pes cavus. Leg length discrepancy was present in 2 of the 45 cases (cases 30 and 31). Less frequent features included cervical stenosis (case 17), spondylolisthesis (case 29), and spina bifida at L2 (case 33). Other musculoskeletal anomalies described in KdVS are fused vertebrae and compression of the spinal cervical cord, ${ }^{13}$ recurrent dislocation of the elbow, patellar dislocation, limited elbow extension and leg length discrepancy requiring femoral osteotomy, and club feet. ${ }^{16,22,26}$ Finally, DornellesWawruk et $a l^{17}$ described a case with chronic anaemia, mild cervical vertebral arthrosis, and multiple thoracic and lumbar vertebral fusions. 


\section{Visual and hearing impairments}

Hypermetropia is present in $38 \%$ of our cohort, and strabismus is noted in $41 \%$. Some other clinical features involving the eye were also present. Case 32, a 43-year-old male with a 17q21.31 deletion, had optic nerve hypoplasia. Wright et al, ${ }^{26}$ reported a similar case with optic nerve hypoplasia and early cataract. Case 29 had cerebral visual impairment (CVI) probably due to neonatal asphyxia and hard-totreat seizures. Delayed visual maturation and mild CVI was also present in case 36, a 5-year-old boy with a KANSL1 SNV and West syndrome, and in cases 38 and 41, both with a KANSL1 SNV and epilepsy. Cataract was not present in our series but has been reported in the literature, ${ }^{13,17}$ as well as coloboma. ${ }^{21}$

Hearing impairment $(25 \%)$ is conductive in most cases and often the result of recurrent otitis media. This has also been described by Tan el al. ${ }^{22}$ Notably, Dubourg et $a l^{18}$ and Wright et $a l^{26}$ described patients with sensorineural hearing loss.

\section{Cardiovascular defects}

The congenital heart anomalies in our series (39\%) mainly included atrial septal defect (ASD) or ventricular septal defect (VSD), but other cardiac anomalies were seen, including patent ductus arteriosus, hypoplastic aortic valve, bicuspid aortic valve, common left pulmonary vein, dysplastic pulmonary valve, dilated left ventricle, anomalous right subclavian artery, doubly committed VSD, broad aorta, patent foramen ovale, and mitral insufficiency. Persistent ductus arteriosus has been reported previously ${ }^{13}$ as well as pulmonary stenosis, intracardiac non-obstructive lesions, bicuspid aortic valve, and a dilated aortic root. $^{18,22,26}$

\section{Renal and urogenital anomalies}

Renal and urogenital anomalies were present in 45\%. Cryptorchidism was present in $53 \%$ of the male patients. Other important anomalies of the renal and urogenital tract included vesiculoureteral reflux, hydronephrosis, pyelectasia, a duplex renal system, a pear-shaped bladder, and macroorchidism. A duplex kidney has been reported in the medical literature twice, but cryptorchidism, hydronephrosis, and vesiculoureteral reflux were the most prevalent features. ${ }^{13,22}$ Less common features included pyelectasia, reflux nephropathy associated hypertension, hypospadia, shawl scrotum, phimosis, and multicystic renal dysplasia. ${ }^{13,18,22}$

\section{Ectodermal abnormalities}

Ectodermal abnormalities were present in $67 \%$ of our cases and have previously been described. ${ }^{13,18,22,24,26,30}$ Skin involvement included, among others, multiple nevi, depigmentosa, hyperkeratosis, eczema, keratosis pilaris, café-au-lait maculae, ichthyosis vulgaris, acne vulgaris, piezogenic papules, and hemangiomas. Importantly, case 17 developed melanoma at 9 years of age. Cases 25 and 32 both had vitiligo. Case 25 has been described previously by Maley et $a l^{31}$ in addition to another child with a 17q21.31 deletion and vitiligo. Hypopigmented cutaneous patches which resemble vitiligo have been described before, ${ }^{16}$ as well as skin changes reminiscent of those seen in patients with cardiofaciocutaneous syndrome (OMIM \#115150). ${ }^{26}$ Other ectodermal abnormalities included abnormal hair color/texture, alopecia, and brittle nails. Dental problems consisted of enamel hypoplasia, caries, absence of secondary elements, and small, widely spaced, or conical teeth.

\section{Miscellaneous}

Case 14, an 8-year-old boy with a 17q21.31 deletion, was born at 37 weeks of gestation with a haemorrhagic parenchymal infarction.
Prenatal ischemic infarction has been described twice, ${ }^{13,18}$ and Vlckova et $a l^{24}$ described a monozygotic twin with KdVS who had perinatal hypoxia during delivery followed by intracranial hemorrhage. ${ }^{24}$ Case 25 had vitiligo and developed Addison's disease with GAD65 autoantibodies. This case has been described in more detail by Maley et al. ${ }^{31}$ Other clinical features that might be of importance are hypertension (case 20), neutropenia (cases 7 and 25), and recurrent infections in at least 11 out of 45 cases (24\%).

\section{PREVALENCE}

In 2008, we estimated the prevalence of the KdVS to be 1 in 16000 individuals. ${ }^{13}$ This was based on 19 cases that had been included in broader cohorts, including a total of 2978 patients with ID (frequency $0.64 \%$ ). This estimation was biased towards deletion positive cohorts. Vulto van Silfhout et $a b^{37}$ report on 10 children with a $17 \mathrm{q} 21.31$ deletion among a cohort of 5531 children with developmental delay and/or ID, giving a frequency of $0.11 \%$ among individuals with unexplained ID, and subsequently, a prevalence of the 17q21.31 deletion of 1 in 55000 individuals. This is in line with the study of Coe et $a l,{ }^{34}$ in which 31 cases were identified in a cohort of 29085 children with developmental delay, ID, and autism spectrum disorder (prevalence of $0.11 \%$ ). In the same cohort, similar numbers were found for Prader Willi syndrome $(\# 176270 ; n=40)$ and Smith Magenis syndrome $(\# 182290 ; n=24) .{ }^{34}$

The prevalence of the KANSL1 SNV cannot be ascertained with precision owing to the limited number of cases identified thus far. In the first 1133 children with developmental disorders in the DDD project (www.ddduk.org/), one de novo KANSL1 variant was identified, giving a frequency of $0.09 \% .{ }^{33}$ Coe et a ${ }^{34}$ report on 7 KANSL1 SNVs among 4716 cases with unexplained developmental delay or autism resulting in a frequency of $0.15 \%$ (although at least one of the mutations was inherited from a non-affected mother, see the section 'Genetic testing'). These data suggest that the pathogenic KANSL1 SNVs might be as frequent as the deletion, but more studies are needed to determine an unbiased prevalence of the syndrome.

\section{DIFFERENTIAL DIAGNOSIS}

In the majority of cases, the diagnosis of KdVS results from genomewide molecular cytogenetic analysis, such as genomic microarrays or whole-exome sequencing. Most findings in KdVS are common and relatively nonspecific indications for genetic analysis. However, the concurrent finding of (severe) neonatal hypotonia, ID with speech that is particularly affected, epilepsy, ASD/VSD, renal or urologic anomalies, cryptorchidism, hypermetropia, and the characteristic facial dysmorphic features is suggestive for the diagnosis of KdVS. Because of the hypotonia, Down syndrome and Prader Willi syndrome may have been considered in the neonatal period. In contrast to Prader Willi syndrome, however, childhood hyperphagia and central obesity have not been reported in KdVS. In some cases with IUGR and short stature, Silver Russell syndrome (\#180860) had been suggested and other children with KdVS have previously been tested for Angelman syndrome (\#105830) because of the ID, epilepsy, and severe speech delay. Moreover, phenotypic similarities of individuals with KdVS to individuals with cardiofaciocutaneous syndrome (\#115150) have been described, including nevi and other pigmentary skin abnormalities, together with ID, a coarse facial appearance, and relative macrocephaly. ${ }^{26}$ The facial dysmorphism might also reflect Ohdo syndrome (\#300895; short palpebral fissures), 22q11 microdeletion syndrome (\#192430; long face, short palpebral fissures, prominent nose), and Fragile X syndrome (\#300624; long face that coarsens with age). 


\section{CONCLUSION}

This study shows that haploinsufficiency of KANSL1 by itself is sufficient to cause the full KdVS phenotype, ${ }^{5,20}$ including neonatal hypotonia, feeding difficulties, developmental delay, ID with speech delay, seizures, characteristic facial dysmorphism, musculoskeletal anomalies, congenital heart defects, renal and urogenital anomalies, and ectodermal anomalies. Although many individuals with KdVS display a social and friendly behaviour, dysfunctional neuropsychological problems are also common.

All KANSL1 SNVs reported so far are truncating variants. Genetic testing of the 17q21.31 locus can be challenging, because of the structural complexity of the genomic region. Especially for a variant in the $5^{\prime}$-end of the KANSL1 gene, analysis of the parental samples is of utmost importance to ensure that the variant is 'de novo', before declared a pathogenic variant.

So far, little is known regarding the natural history of KdVS. Therefore, further clinical studies (www.17q21.com) including longitudinal cognitive-socio-behavioural studies are needed to provide proper counselling to parents and caregivers. Moreover, functional studies (eg, using induced pluripotent stem cells and animal models) will give more insights in the molecular mechanisms by which haploinsufficiency of KANSL1 produces the broad spectrum of clinical features.

\section{CONFLICT OF INTEREST}

The authors declare no conflict of interest.

\section{ACKNOWLEDGEMENTS}

We thank the individuals and their parents for participation in this study. We are especially thankful to the board members of the foundation Supporting Families with Koolen-de Vries Syndrome (http://www.supportingkdvs.com/). We also thank T Dijkhuizen for sharing microarray data with us and L van Diepen, JM Geelen, WM Wissink-Lindhout, and KJ Pijnenburg-Kleizen for data management. The study was financially supported by the Netherlands Organisation for Health Research and Development (ZON-MW grant 917-86319 and 912-12-109 to BBAdV) and by the Italian Ministry of Health and ' 5 per mille' funding (to PF, CB, EA, CR). JG was supported by Australian NHMRC grant 628952 and Research Fellowship 1041920. The DDD Study is funded by HICF-1009-003 and WT098051. This study uses data from the 1000 Genomes project phase 3 data release.

1 Koolen DA, Vissers LE, Pfundt R et al: A new chromosome 17q21.31 microdeletion syndrome associated with a common inversion polymorphism. Nat Genet 2006; 38 999-1001.

2 Shaw-Smith C, Pittman AM, Willatt $\mathrm{L}$ et al: Microdeletion encompassing MAPT at chromosome $17 q 21.3$ is associated with developmental delay and learning disability. Nat Genet 2006; 38: 1032-1037.

3 Sharp AJ, Hansen S, Selzer RR et al: Discovery of previously unidentified genomic disorders from the duplication architecture of the human genome. Nat Genet 2006; 38: 1038-1042.

4 Koolen DA, Kramer JM, Neveling $\mathrm{K}$ et al: Mutations in the chromatin modifier gene KANSL1 cause the 17q21.31 microdeletion syndrome. Nat Genet 2012; 44: 639-641.

5 Zollino M, Orteschi D, Murdolo $\mathrm{M}$ et al: Mutations in KANSL1 cause the 17q21.31 microdeletion syndrome phenotype. Nat Genet 2012; 44: 636-638.

6 Dias J, Van Nguyen N, Georgiev P et al: Structural analysis of the KANSL1MDR5/ KANSL2 complex reveals that WDR5 is required for efficient assembly and chromatin targeting of the NSL complex. Genes Dev 2014; 28: 929-942.

7 Cai Y, Jin J, Swanson SK et al: Subunit composition and substrate specificity of a MOF-containing histone acetyltransferase distinct from the male-specific lethal (MSL) complex. J Biol Chem 2010; 285: 4268-4272.
8 Raja SJ, Charapitsa I, Conrad T et al: The nonspecific lethal complex is a transcriptional regulator in Drosophila. Mol Cell 2010; 38: 827-841.

9 Mendjan S, Taipale M, Kind J et al: Nuclear pore components are involved in the transcriptional regulation of dosage compensation in Drosophila. Mol Cell 2006; 21: 811-823.

10 Steinberg KM, Antonacci F, Sudmant PH et al: Structural diversity and African origin of the 17q21.31 inversion polymorphism. Nat Genet 2012; 44: 872-880.

11 Boettger LM, Handsaker RE, Zody MC, McCarroll SA: Structural haplotypes and recent evolution of the human 17q21.31 region. Nat Genet 2012; 44: 881-885.

12 Stefansson $\mathrm{H}$, Helgason A, Thorleifsson $\mathrm{G}$ et al: A common inversion under selection in Europeans. Nat Genet 2005; 37: 129-137.

13 Koolen DA, Sharp AJ, Hurst JA et al: Clinical and molecular delineation of the 17q21.31 microdeletion syndrome. J Med Genet 2008; 45: 710-720.

14 Zody MC, Jiang Z, Fung HC et al: Evolutionary toggling of the MAPT 17q21.31 inversion region. Nat Genet 2008; 40: 1076-1083.

15 Itsara A, Vissers LE, Steinberg KM et al: Resolving the breakpoints of the 17q21.31 microdeletion syndrome with next-generation sequencing. Am J Hum Genet 2012; 90 599-613.

16 Digilio MC, Bernardini L, Capolino R et al: Hypopigmented skin patches in 17q21.31 microdeletion syndrome: expanding the spectrum of cutaneous findings. Clin Dysmorphol 2014; 23: 32-34.

17 Dornelles-Wawruk H, Pic-Taylor A, Rosenberg C et al: Complex phenotype associated with 17q21.31 microdeletion. Mol Syndromol 2013; 4: 297-301.

18 Dubourg C, Sanlaville D, Doco-Fenzy M et al: Clinical and molecular characterization of $17 q 21.31$ microdeletion syndrome in 14 French patients with mental retardation. Eur J Med Genet 2011; 54: 144-151.

19 El Chehadeh-Djebbar S, Callier P, Masurel-Paulet A et al: 17q21.31 microdeletion in a patient with pituitary stalk interruption syndrome. Eur J Med Genet 2011; 54: 369-373.

20 Koolen DA, Dupont J, de Leeuw $\mathrm{N}$ et al: Two families with sibling recurrence of the 17q21.31 microdeletion syndrome due to low-grade mosaicism. Eur J Hum Genet 2012; 20: 729-733.

21 Sharkey FH, Morrison N, Murray R et al: 17q21.31 microdeletion syndrome: further expanding the clinical phenotype. Cytogenet Genome Res 2009; 127: 61-66.

22 Tan TY, Aftimos S, Worgan L et al: Phenotypic expansion and further characterisation of the 17q21.31 microdeletion syndrome. J Med Genet 2009; 46: 480-489.

23 Terrone G, D'Amico A, Imperati F et al: A further contribution to the delineation of the 17q21.31 microdeletion syndrome: central nervous involvement in two Italian patients. Eur J Med Genet 2012; 55: 466-471.

24 Vlckova M, Hancarova M, Drabova J et al: Monozygotic twins with 17q21.31 microdeletion syndrome. Twin Res Hum Genet 2014; 17: 405-410.

25 Wray CD: 17q21.31 microdeletion associated with infantile spasms. Eur J Med Genet 2013; 56: 59-61.

26 Wright EB, Donnai D, Johnson D, Clayton-Smith J: Cutaneous features in 17q21.31 deletion syndrome: a differential diagnosis for cardio-facio-cutaneous syndrome. Clin Dysmorphol 2011; 20: 15-20.

27 Varela MC, Krepischi-Santos AC, Paz JA et al: A 17q21.31 microdeletion encompassing the MAPT gene in a mentally impaired patient. Cytogenet Gen Res 2006; 114: 89-92.

28 Cooper GM, Coe BP, Girirajan S et al: A copy number variation morbidity map of developmental delay. Nat Genet 2011; 43: 838-846.

29 Jaillard S, Drunat S, Bendavid C et al: Identification of gene copy number variations in patients with mental retardation using array-CGH: novel syndromes in a large French series. Eur J Med Genet 2010; 53: 66-75.

30 Kitsiou-Tzeli S, Frysira H, Giannikou $\mathrm{K}$ et al: Microdeletion and microduplication $17 q 21.31$ plus an additional CNV, in patients with intellectual disability, identified by array-CGH. Gene 2012; 492: 319-324.

31 Maley AM, Spraker MK, de Vries BB, Koolen DA: Vitiligo in the Koolen-de Vries or 17q21.31 microdeletion syndrome. Clin Dysmorphol 2015; 24: 86-87.

32 Barone C, Novelli A, Capalbo A et al: An additional clinical sign of 17q21.31 microdeletion syndrome: preaxial polydactyly of hands with broad thumbs. Am J Med Genet Part A 2015; 167: 1671-1673.

33 Wright CF, Fitzgerald TW, Jones WD et al: Genetic diagnosis of developmental disorders in the DDD study: a scalable analysis of genome-wide research data. Lancet 2014; 385: 1305-1314.

34 Coe BP, Witherspoon K, Rosenfeld JA et al: Refining analyses of copy number variation identifies specific genes associated with developmental delay. Nat Genet 2014; 46 : 1063-1071.

35 Fokkema IF, Taschner PE, Schaafsma GC, Celli J, Laros JF, den Dunnen JT: LOVD v.2.0: the next generation in gene variant databases. Hum Mutat 2011; 32 557-563.

361000 Genomes Project Consortium, Abecasis GR, Auton A, Brooks LD et al: An integrated map of genetic variation from 1,092 human genomes. Nature 2012; 491: $56-65$.

37 Vulto-van Silfhout AT, Hehir-Kwa JY, van Bon BW et al: Clinical significance of de novo and inherited copy-number variation. Hum Mutat 2013; 34: 1679-1687.

Supplementary Information accompanies this paper on European Journal of Human Genetics website (http://www.nature.com/ejhg) 